\title{
Chandra Observation of the AKARI NEP Deep Field
}

\author{
Takamitsu Miyaji, M. Krumpe, H. Brunner \\ and AKARI NEP Deep Survey Team
}

Inst. de Astron. sede Ensenada, Univ. Nacional Autonoma de Mexico and UCSD CASS, Mexico/USA

E-mail: miyaji@astrosen.unam.mx

\begin{abstract}
The AKARI NEP Deep Field Survey is an international multiwavelength survey over $0.4 \mathrm{deg}^{2}$ of the sky. This is the deepest survey made by the InfraRed Camera (IRC) of the infrared astronomical satellite AKARI with 9 filters continuously covering the 2-25 $\mu \mathrm{m}$ range. This has been supplemented by other ground-based and space multiwavelength data ranging from X-ray (Chandra), UV (GALEX), Optical-NIR (Subaru Sprime-cam, CFHT/WIRCAM, CFHT/Megacam, KPNO Flamningos among others for imagings as well as Keck Diemos, Subaru Focas, Subaru FMOS, WIYN Hydra, and GTC OSIRIS for spectra), far-infrared (Herschel) and radio (WSRT and e-Merlin). The uniqueness of the field lies in the availability of four filters between 9-18 $\mu \mathrm{m}$, which fall into the Spitzer gap between the IRAC and MIPS instruments. This made this field one of the deepest at $\sim 15 \mu \mathrm{m}$ and the deepest among those with similar solid angles. This enabled us to make sensitive MIR detection of AGN candidates around $\mathrm{z}$ $\sim 1$. The MIR selection is based on hot dust emission in the AGN torus and is efficient in detecting highly obscured Compton-thick AGN population. A number of team members have worked (e.g. Hanami et al. 2012) or are working on a catalog of AGN candidates in this field. In this presentation, we report the results of the Chandra observations on this field. The field was covered by 15 overlapping Chandra ACIS-I observations (including our own and from archive) with a total exposure of $310 \mathrm{ks}$, detecting $\sim 500 \mathrm{X}$-ray sources. We explain our improved source detection procedure for highly overlapped Chandra images and results. We utilize the stacking analysis (both in the observed and rest-frame) of the MIR AGN candidates that are not detected individually. The stacking analysis is expected to detect the summed X-ray flux from scattered components and Fe-lines. The results are discussed in terms of quantifying the Compton-thick populations at $\mathrm{z} \sim 1$.
\end{abstract}

